\title{
High-spin isomeric structures in exotic odd-odd nuclei: Exploration of the proton drip line and beyond
}

\author{
H. L. Liu, ${ }^{1}$ F. R. Xu, ${ }^{1,2, *}$ S. W. Xu, ${ }^{3}$ R. Wyss, ${ }^{4}$ and P. M. Walker ${ }^{5}$ \\ ${ }^{1}$ State Key Laboratory of Nuclear Physics and Technology, School of Physics, Peking University, Beijing 100871, People’s Republic of China \\ ${ }^{2}$ Center for Theoretical Nuclear Physics, National Laboratory for Heavy Ion Physics, Lanzhou 730000, People's Republic of China \\ ${ }^{3}$ Institute of Modern Physics, Chinese Academy of Sciences, Lanzhou 730000, People's Republic of China \\ ${ }^{4}$ AlbaNova University Center, KTH (Royal Institute of Technology), S-106 91 Stockholm, Sweden \\ ${ }^{5}$ Department of Physics, University of Surrey, Guildford, Surrey GU2 7XH, United Kingdom
}

(Received 31 May 2007; published 11 September 2007)

\begin{abstract}
We investigate the role of two-quasiparticle isomeric states along the proton drip line, using configurationconstrained potential-energy-surface calculations. In contrast to even-even nuclei, odd-odd nuclei can have coexisting low-lying two-quasiparticle states. The low excitation energy and high angular momentum can lead to long-lived isomers. Also, because of the hindrance by spin selection, the probabilities of $\beta$ and proton decays from high-spin isomers can be reduced significantly. The present calculations reproduce reasonably well the available data for observed isomers in such nuclei. Unobserved high-spin isomers are predicted, which could be useful for future experimental studies of exotic nuclei at and beyond the proton drip line.
\end{abstract}

DOI: 10.1103/PhysRevC.76.034313

PACS number(s): 21.10.-k, 21.60.-n, 23.20.Lv

\section{INTRODUCTION}

Exotic nuclei with extreme ratios of neutron and proton numbers usually have very short lifetimes. Thanks to the advance of radioactive nuclear beam (RNB) techniques, more and more exotic nuclei far from the valley of $\beta$ stability are being probed. However, there are still predicted to exist many as-yet unobserved nuclei, where weak binding may lead to new physics with important impact on astrophysical nucleosynthesis processes [1]. Access to and beyond the proton drip line is achieved usually through fusion-evaporation reactions. By comparison, neutron-drip-line nuclei are more difficult to reach, and new techniques are required.

For exotic nuclei, isomeric states, by virtue of their long lifetimes, can play an important role in their experimental investigation (see, e.g., Refs. [2,3]). Indeed, it has been pointed out that the stability of superheavy nuclei can be increased due to high-spin isomerism [4]. The influence of high-spin isomers on even-even drip-line nuclei has also been seen, for example, in the proton-drip-line nucleus ${ }^{140} \mathrm{Dy}$ with an $8^{-}$isomer observed [5,6]. In even-even nuclei, multiquasiparticle excitations involve breaking one or more pairs of nucleons, which results in significant excitation energies. In principle, the stability of a state decreases with increasing excitation energy. However, the situation is different in odd-odd nuclei in which the ground state corresponds to a two-quasiparticle (2qp) configuration. As a consequence, long-lived isomers can frequently be found in odd-odd nuclei. Indeed, high-spin isomers with remarkably long lifetimes have been observed in a variety of exotic odd-odd nuclei [7].

In deformed nuclei, the angular-momentum projections (onto the symmetry axis) of the odd proton $\left(\Omega_{p}\right)$ and the odd neutron $\left(\Omega_{n}\right)$ couple to a total spin projection, $K$, with a well-established Gallagher-Moszkowski (GM) [8] splitting

\footnotetext{
*frxu@pku.edu.cn
}

of the doublet states with $K=\left|\Omega_{p}+\Omega_{n}\right|$ and $\left|\Omega_{p}-\Omega_{n}\right|$ in odd-odd nuclei [9]. For the present purposes, the situation when both the odd neutron and the odd proton occupy high- $\Omega$ orbitals is particularly interesting, because it leads to high-spin and low-spin states at similar energies. Since electromagnetic transitions from high-spin to low-spin states are strongly hindered, low-lying high-spin states in exotic nuclei can be forced to decay through $\beta$ radioactivity. High-spin states in nuclei at and beyond the proton drip line can decay by direct proton emission. The large spin change can also have significant influence on the $\beta$ and proton decay rates, which will be discussed later. In the present work, systematic calculations are used to identify candidates for long-lived isomers close to the proton drip line.

\section{THE MODEL}

Configuration-constrained potential-energy-surface (PES) calculations [10] have been performed to determine the deformations and excitation energies of quasiparticle states. Single-particle levels are obtained from the nonaxial deformed Woods-Saxon potential with the set of universal parameters [11]. In the pairing treatment, particle-number projection is approximated by the Lipkin-Nogami method [12]. In the configuration-constrained PES calculation, it is required to adiabatically block the unpaired nucleon orbitals that specify a given configuration. This has been achieved by calculating and identifying the average Nilsson quantum numbers for every orbital involved in the configuration [10]. The total energy of a state consists of a macroscopic part that is obtained with the standard liquid-drop model [13] and a microscopic part that is calculated by the Strutinsky shell-correction approach, including blocking effects. The PES is calculated in the space of quadrupole $\left(\beta_{2}, \gamma\right)$ and hexadecapole $\left(\beta_{4}\right)$ deformations. The configuration-constrained PES calculation can properly treat the shape polarization due to unpaired nucleons. 
The pairing strength $(G)$ is first determined by the average gap method [14] and then adjusted to reproduce the experimental odd-even mass difference using a five-point formula in both experiment and theory as discussed in Ref. [15]. Such an adjustment of the pairing strength is required so as to include the shape and blocking effects on the mass difference [15]. The adjustment does not affect much the excitation energies of 2qp states in odd-odd nuclei, because excited 2qp states and the ground state have the same seniority (i.e., the same number of unpaired nucleons). However, the modification of the pairing strength is important for the calculation of the $\beta$-decay energy, because an odd-odd nucleus is transformed by $\beta$ decay into an even-even nucleus (seniority zero) in which the pairing correlation is stronger. The small adjustments of pairing strengths can result in considerable changes of pairing energies in even-even nuclei $[10,15]$. It will be seen that modified pairing strengths can reproduce well experimental $\beta$-decay energies.

\section{CAlCulations}

Configuration-constrained PES calculations can predict well the properties of quasiparticle states in deformed nuclei, including deformations, energies, and configurations $[4,10]$. We surveyed systematically the possible isomeric states in odd-odd deformed nuclei at the proton-rich limit of current experimental knowledge. Figure 1 shows our predictions for low-lying 2qp high-spin states in deformed odd-odd nuclei. These high-spin states are formed by the spin coupling of the odd neutron and the odd proton. Due to no extra pair breaking compared to ground states, the high-spin 2qp states can have very low excitation energies, sometimes even becoming ground states. Figure 1 shows that predicted high-spin 2qp states mainly locate in the $A \sim 70,130,190$, and transuranium regions. Later, we will discuss in more detail the $A \sim 130$ nuclei where more experimental observations are available.

In the mass 70 region, calculated ground-state PESs show prolate-oblate shape coexistence. Particularly for those nuclei near the center of the region (i.e., both $N$ and $Z$ are around 35) oblate minima lie lower than prolate minima. Our calculations give $\beta_{2} \approx-0.3$ oblate states in the $A \sim 70$ nuclei. At such an oblate deformation, the Fermi surfaces of the $A \sim 70$ nuclei locate around the high- $\Omega$ single-particle orbitals of the $g_{9 / 2}$ subshell. Indeed, a $9^{+}$isomer in ${ }^{70} \mathrm{Br}$ has been observed with the configuration of $\pi 9 / 2^{+}[404] \otimes \nu 9 / 2^{+}$[404] [16]. Despite being at a high excitation energy of $2.23 \mathrm{MeV}$ [17], the isomer has a half-life of $2.2 \mathrm{~s}$ [16], much longer than the $79 \mathrm{~ms}$ of the $0^{+}$ground state. The present calculation shows that the $9^{+}$isomer has a $\beta_{2}=-0.28$ oblate shape and an excitation energy of $2.56 \mathrm{MeV}$, in good agreement with the measured energy. The experiment [16] observed that the $9^{+}$isomer has direct $\beta^{+} /$EC decay into the $8^{+}$or $9^{+}$state of the daughter nucleus ${ }^{70} \mathrm{Se}$. For the isomer, the $\gamma$ decay is highly hindered because of the high multipolarities needed for decay into the lower-energy, low-spin states. It is worth noting that the isomer has a $\beta$-decay energy lower than that of the ground state [17]. This is because a high-spin 2qp state of an odd-odd nucleus decays into a high-spin state of the even-even daughter, due to the spin selection in $\beta$ decay. The lower $\beta$-decay energy leads to a longer $\beta$-decay lifetime. For $\beta^{+}$decay, the lifetime is inversely proportional to the fifth power of the decay energy according to the Sargent rule [18].

Long-lived high-spin 2qp isomers appear also in $A \sim 130$ odd-odd nuclei. In Table I, we list our calculations of low-lying 2qp states in odd-odd Cs and La isotopes. In the Cs isotopes, long-lived high-spin isomers have been observed [7]. For the La isotopes, we give predictions of possible low-lying, high-spin isomers. In these odd-odd isotopes, calculations show that there are several low-lying, low-spin states with excitation energies less than $100 \mathrm{keV}$ (see Table I). In these cases, it is difficult to judge which configurations are ground states, because we should not expect that the model is able to give energies with accuracy better than several tens of $\mathrm{keV}$. In the $A \sim 130$ mass region, high-spin states are formed because of the occupation of the proton $9 / 2^{+}[404]$ orbital. In ${ }^{116} \mathrm{Cs}$, for example, the observed isomer has a half-life of $3.9 \mathrm{~s}$,

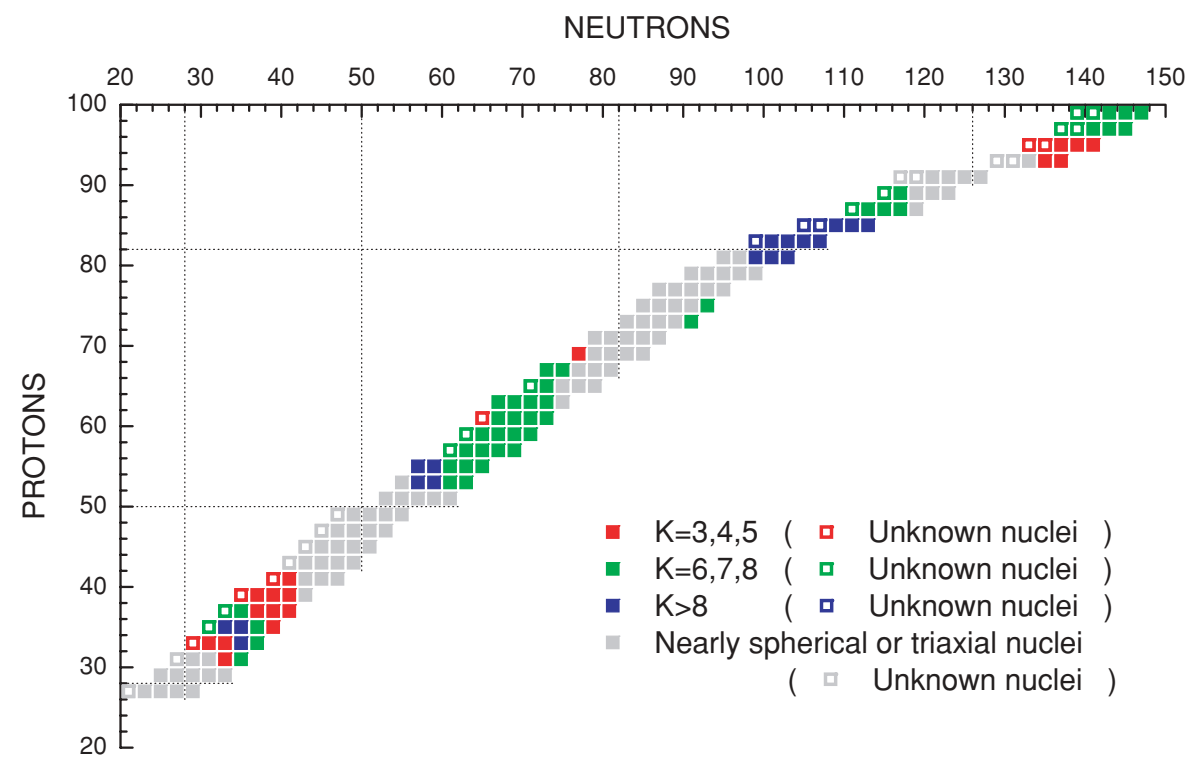

FIG. 1. (Color online) Predicted high-spin states in odd-odd nuclei at the proton-rich limit of current experimental knowledge. 
TABLE I. Calculations of low-lying 2qp states in $Z=55,57$ isotopes compared with available experimental data [7,19].

\begin{tabular}{|c|c|c|c|c|c|c|c|c|c|}
\hline \multirow[t]{2}{*}{ Nuclei } & \multicolumn{5}{|c|}{ Calculations $^{\mathrm{a}}$} & \multicolumn{4}{|c|}{ Experiments $^{\mathrm{b}}[7,19]$} \\
\hline & Configurations & $J^{\pi}$ & $\beta_{2}$ & $E_{x}(\mathrm{keV})$ & $Q_{\mathrm{EC}}(\mathrm{MeV})$ & $J^{\pi}$ & $E_{x}(\mathrm{keV})$ & $Q_{\mathrm{EC}}(\mathrm{MeV})$ & $T_{1 / 2}$ \\
\hline \multirow[t]{4}{*}{${ }^{116} \mathrm{Cs}_{61}$} & $\pi \frac{3}{2}[422] \otimes v \frac{3}{2}[411]$ & $0^{+}\left(3^{+}\right)$ & 0.24 & 0 & 11.312 & $\left(1^{+}\right)$ & 0 & $10.980^{\#}$ & $700 \mathrm{~ms}$ \\
\hline & $\pi \frac{1}{2}[420] \otimes v \frac{3}{2}[411]$ & $2^{+}\left(1^{+}\right)$ & 0.24 & 12 & & & & & \\
\hline & $\pi \frac{1}{2}[550] \otimes v \frac{3}{2}[411]$ & $2^{-}\left(1^{-}\right)$ & 0.25 & 17 & & & & & \\
\hline & $\pi \frac{9}{2}[404] \otimes v \frac{3}{2}[411]$ & $6^{+}\left(3^{+}\right)$ & 0.28 & 293 & & $\left(4^{+}, 5,6\right)$ & $100 \pm 60^{\#}$ & & $3.85 \mathrm{~s}$ \\
\hline \multirow[t]{4}{*}{${ }^{118} \mathrm{Cs}_{63}$} & $\pi \frac{3}{2}[422] \otimes v \frac{5}{2}[532]$ & $1^{-}\left(4^{-}\right)$ & 0.25 & 0 & 9.878 & 2 & 0 & 9.670 & $14 \mathrm{~s}$ \\
\hline & $\pi \frac{1}{2}[550] \otimes \nu \frac{5}{2}[532]$ & $3^{+}\left(2^{+}\right)$ & 0.26 & 9 & & & & & \\
\hline & $\pi \frac{1}{2}[420] \otimes \nu \frac{5}{2}[532]$ & $3^{-}\left(2^{-}\right)$ & 0.26 & 27 & & & & & \\
\hline & $\pi \frac{9}{2}[404] \otimes v \frac{5}{2}[532]$ & $7^{-}\left(2^{-}\right)$ & 0.28 & 161 & & $\left(7^{-}\right)$ & $100 \pm 60^{\#}$ & & $17 \mathrm{~s}$ \\
\hline \multirow[t]{5}{*}{${ }^{120} \mathrm{Cs}_{65}$} & $\pi \frac{3}{2}[422] \otimes v \frac{5}{2}[413]$ & $4^{+}\left(1^{+}\right)$ & 0.25 & 0 & 8.391 & $2^{\left(-^{\#}\right)}$ & 0 & 8.284 & $61.2 \mathrm{~s}$ \\
\hline & $\pi \frac{3}{2}[422] \otimes v \frac{5}{2}[532]$ & $1^{-}\left(4^{-}\right)$ & 0.24 & 22 & & & & & \\
\hline & $\pi \frac{1}{2}[420] \otimes v \frac{5}{2}[413]$ & $2^{+}\left(3^{+}\right)$ & 0.25 & 68 & & & & & \\
\hline & $\pi \frac{1}{2}[550] \otimes v \frac{5}{2}[413]$ & $2^{-}\left(3^{-}\right)$ & 0.26 & 80 & & & & & \\
\hline & $\pi \frac{9}{2}[404] \otimes v \frac{5}{2}[532]$ & $7^{-}\left(2^{-}\right)$ & 0.27 & 327 & & $\left(7^{-}\right)$ & $100 \pm 60^{\#}$ & & $57 \mathrm{~s}$ \\
\hline \multirow[t]{3}{*}{${ }^{118} \mathrm{La}_{61}$} & $\pi \frac{3}{2}[541] \otimes \nu \frac{5}{2}[532]$ & $4^{+}\left(1^{+}\right)$ & 0.30 & 0 & 12.891 & & & $12.750^{\#}$ & $200^{\#} \mathrm{~ms}$ \\
\hline & $\pi \frac{3}{2}[422] \otimes \nu \frac{5}{2}[532]$ & $1^{-}\left(4^{-}\right)$ & 0.30 & 11 & & & & & \\
\hline & $\pi \frac{9}{2}[404] \otimes \nu \frac{5}{2}[532]$ & $7^{-}\left(2^{-}\right)$ & 0.32 & 105 & & & & & \\
\hline \multirow[t]{3}{*}{${ }^{120} \mathrm{La}_{63}$} & $\pi \frac{3}{2}[541] \otimes v \frac{3}{2}[411]$ & $3^{-}\left(0^{-}\right)$ & 0.30 & 0 & 11.390 & & 0 & $11.200^{\#}$ & $2.8 \mathrm{~s}$ \\
\hline & $\pi \frac{3}{2}[422] \otimes v \frac{3}{2}[411]$ & $0^{+}\left(3^{+}\right)$ & 0.30 & 34 & & & & & \\
\hline & $\pi \frac{9}{2}[404] \otimes \nu \frac{3}{2}[411]$ & $6^{+}\left(3^{+}\right)$ & 0.32 & 92 & & & & & \\
\hline \multirow[t]{3}{*}{${ }^{122} \mathrm{La}_{65}$} & $\pi \frac{3}{2}[541] \otimes v \frac{5}{2}[413]$ & $1^{-}\left(4^{-}\right)$ & 0.29 & 0 & 9.951 & & 0 & $10.070^{\#}$ & $8.7 \mathrm{~s}$ \\
\hline & $\pi \frac{3}{2}[422] \otimes v \frac{5}{2}[413]$ & $4^{+}\left(1^{+}\right)$ & 0.29 & 34 & & & & & \\
\hline & $\pi \frac{9}{2}[404] \otimes \nu \frac{5}{2}[532]$ & $7^{-}\left(2^{-}\right)$ & 0.30 & 370 & & & & & \\
\hline
\end{tabular}

${ }^{a}$ The theoretical spin assignments in parentheses are for energetically unfavored states according to the GM rule.

${ }^{\mathrm{b}}$ The pound symbol indicates estimated values from systematics, and the parentheses indicate the ambiguity in the experimental assignments of spins and parities.

which is longer than the ground-state half-life of $700 \mathrm{~ms}$ [7]. The experiment has not pinned down the spin and parity of the observed isomer [7]. Our calculation suggests the $J^{\pi}=$ $6^{+}, \pi 9 / 2^{+}[404] \otimes v 3 / 2^{+}[411]$ configuration for the isomer. Similar to the $9^{+}$isomer in ${ }^{70} \mathrm{Br}$, the $6^{+}$isomer in ${ }^{116} \mathrm{Cs}$ was found to $\beta^{+} / \mathrm{EC}$ decay with decay energy lower than that of the low-spin ground state [20]. As mentioned above, the lower $\beta$-decay energy contributes to the longer lifetime. The $\beta_{2}$ deformation of the isomer is calculated to be 0.28 , which is larger than the 0.24 of the ground state and the 0.22 of the daughter nucleus ${ }^{116} \mathrm{Xe}$. The shape polarization is due to the occupation of the proton $9 / 2^{+}$[404] orbital. This polarization also happens for other $\mathrm{Cs}$ and $\mathrm{La}$ isomers.

Calculated results for $N=67,69$ isotones are listed in Table II. In ${ }^{122} \mathrm{Cs}$, our calculation gives the configuration $\pi 3 / 2^{+}[422] \otimes v 1 / 2^{+}[411]$ for the ground state. This is consistent with the experimental assignment [26]. As pointed out in Ref. [26], the configuration coupling in the $1^{+}$state violates the GM rule. In Table II, the states with the $J^{\pi}=$ $8^{-}\left(\pi 9 / 2^{+}[404] \otimes \nu 7 / 2^{-}[523]\right)$ configuration are particularly interesting because of the very high spin value. In ${ }^{122} \mathrm{Cs}$, the $8^{-}$isomer has been observed [7] with a half-life of $3.7 \mathrm{~m}$, which is much longer than the $21 \mathrm{~s}$ of the $1^{+}$ground state [7]. The calculation gives $\beta_{2}=0.27$ for the observed $8^{-}$isomer and reproduces well the experimental $\beta_{2}$ difference $\left(\Delta \beta_{2} \approx 0.03\right.$ ) between the $8^{-}$isomer and $1^{+}$ground state [27]. From our calculations, $J^{\pi}=8^{-}$states appear also in ${ }^{124} \mathrm{La}$ and ${ }^{126} \mathrm{La}$, with properties similar to those of the $8^{-}$isomer in ${ }^{122} \mathrm{Cs}$. Indeed, a long-lived isomer with a possible experimental assignment of $7^{-}$or $8^{-}$was observed in ${ }^{124} \mathrm{La} \mathrm{[7].} \mathrm{In}{ }^{126} \mathrm{La}$, experiments have not seen the $8^{-}$isomer, but did observe low-lying isomers with possible spins $\leqslant 5$ [23]. In $\mathrm{Pr}$ and $\mathrm{Pm}$ isotopes, experiments have seen the high-spin isomeric states $[7,25]$ without the low-spin states being observed. In ${ }^{128} \mathrm{Pr}$, the calculation gives a $5^{+}$configuration for the ground state (see Table II), which disagrees with the newly assigned $3^{+}$configuration [24], but agrees with the earlier experimental assignment [25]. In ${ }^{130} \mathrm{Pm}$, a recent experiment [28] observed a signal for the existence of a low-spin isomer. Unfortunately, the information is not enough to deduce a clear result. It is possible that the high-spin isomers in Pr and Pm isotopes were observed prior to the low-spin states because of the enhanced stability caused by high-spin isomerism. The high-spin isomers in Table II are formed because of the occupation of the neutron $7 / 2^{-}$[523] orbital, while low-spin states appear with the occupation of the neutron $1 / 2^{+}[411]$ orbital.

As mentioned above, the $\beta$-decay energy is a sensitive probe of the adjustment of the pairing strength. In Ref. [19], 
TABLE II. Same as Table I but for $N=67,69$ isotones.

\begin{tabular}{|c|c|c|c|c|c|c|c|c|c|}
\hline \multirow[t]{2}{*}{ Nuclei } & \multicolumn{5}{|c|}{ Calculations } & \multicolumn{4}{|c|}{ Experiments $[7,19]$} \\
\hline & Configurations & $J^{\pi}$ & $\beta_{2}$ & $E_{x}(\mathrm{keV})$ & $Q_{\mathrm{EC}}(\mathrm{MeV})$ & $J^{\pi}$ & $E_{x}(\mathrm{keV})$ & $Q_{\mathrm{EC}}(\mathrm{MeV})$ & $T_{1 / 2}$ \\
\hline \multirow[t]{3}{*}{${ }^{122} \mathrm{Cs}_{67}$} & $\pi \frac{3}{2}[422] \otimes v \frac{1}{2}[411]$ & $2^{+}\left(1^{+}\right)$ & 0.24 & 0 & 7.284 & $1^{+}$ & 0 & 7.220 & $21.18 \mathrm{~s}$ \\
\hline & $\pi \frac{3}{2}[422] \otimes \nu \frac{7}{2}[523]$ & $2^{-}\left(5^{-}\right)$ & 0.24 & 137 & & & & & \\
\hline & $\pi \frac{9}{2}[404] \otimes \nu \frac{7}{2}[523]$ & $8^{-}\left(1^{-}\right)$ & 0.27 & 328 & & $8^{-}$ & $140 \pm 30$ & & $3.70 \mathrm{~m}$ \\
\hline \multirow[t]{3}{*}{${ }^{124} \mathrm{La}_{67}$} & $\pi \frac{1}{2}[550] \otimes v \frac{1}{2}[411]$ & $0^{-}\left(1^{-}\right)$ & 0.27 & 0 & 8.946 & $\operatorname{low}^{\left(+^{\#}\right)}$ & $100 \pm 100^{\#}$ & & $21 \mathrm{~s}$ \\
\hline & $\pi \frac{1}{2}[550] \otimes v \frac{7}{2}[523]$ & $4^{+}\left(3^{+}\right)$ & 0.27 & 97 & & & & & \\
\hline & $\pi \frac{9}{2}[404] \otimes v \frac{7}{2}[523]$ & $8^{-}\left(1^{-}\right)$ & 0.30 & 284 & & $\left(7^{-}, 8^{-}\right)$ & 0 & 8.830 & $29.21 \mathrm{~s}$ \\
\hline \multirow[t]{2}{*}{${ }^{126} \operatorname{Pr}_{67}$} & $\pi \frac{3}{2}[541] \otimes \nu \frac{1}{2}[411]$ & $1^{-}\left(2^{-}\right)$ & 0.30 & 0 & 10.554 & & & & \\
\hline & $\pi \frac{3}{2}[541] \otimes \nu \frac{7}{2}[523]$ & $5^{+}\left(2^{+}\right)$ & 0.30 & 63 & & $(4,5,6)$ & 0 & $10.560^{\#}$ & $3.12 \mathrm{~s}$ \\
\hline \multirow[t]{2}{*}{${ }^{128} \mathrm{Pm}_{67}$} & $\pi \frac{5}{2}[532] \otimes \nu \frac{1}{2}[411]$ & $2^{-}\left(3^{-}\right)$ & 0.32 & 0 & 12.338 & & & & \\
\hline & $\pi \frac{5}{2}[532] \otimes v \frac{7}{2}[523]$ & $6^{+}\left(1^{+}\right)$ & 0.32 & 21 & & $(5,6,7)[21]$ & 0 & $12.140^{\#}$ & $1.0 \mathrm{~s}$ \\
\hline \multirow[t]{2}{*}{${ }^{130} \mathrm{Eu}_{67}{ }^{\mathrm{a}}$} & $\pi \frac{3}{2}[411] \otimes v \frac{1}{2}[411]$ & $1^{+}\left(2^{+}\right)$ & 0.32 & 0 & & $\left(1^{+}\right)[22]$ & 0 & & $1.1 \mathrm{~ms}$ \\
\hline & $\pi \frac{3}{2}[411] \otimes \nu \frac{7}{2}[523]$ & $5^{-}\left(2^{-}\right)$ & 0.32 & 7 & & & & & \\
\hline \multirow[t]{3}{*}{${ }^{126} \mathrm{La}_{69}$} & $\pi \frac{1}{2}[550] \otimes \nu \frac{7}{2}[523]$ & $4^{+}\left(3^{+}\right)$ & 0.26 & 0 & 7.720 & $(4,5)[23]$ & 0 & 7.700 & $54 \mathrm{~s}$ \\
\hline & $\pi \frac{1}{2}[550] \otimes \nu \frac{1}{2}[411]$ & $0^{-}\left(1^{-}\right)$ & 0.26 & 122 & & $\begin{array}{c}\left(0^{-}, 1^{ \pm}, 2^{-}\right) \\
{[23]}\end{array}$ & $210 \pm 410$ & & $20 \mathrm{~s}$ \\
\hline & $\pi \frac{9}{2}[404] \otimes \nu \frac{7}{2}[523]$ & $8^{-}\left(1^{-}\right)$ & 0.29 & 310 & & & & & \\
\hline \multirow[t]{2}{*}{${ }^{128} \operatorname{Pr}_{69}$} & $\pi \frac{3}{2}[541] \otimes \nu \frac{7}{2}[523]$ & $5^{+}\left(2^{+}\right)$ & 0.29 & 0 & 9.274 & $\begin{array}{l}\left(3^{+}\right)[24] \\
(4,5)[25]\end{array}$ & 0 & 9.200 & $2.84 \mathrm{~s}$ \\
\hline & $\pi \frac{3}{2}[541] \otimes v \frac{1}{2}[411]$ & $1^{-}\left(2^{-}\right)$ & 0.29 & 82 & & & & & \\
\hline \multirow[t]{2}{*}{${ }^{130} \mathrm{Pm}_{69}$} & $\pi \frac{5}{2}[532] \otimes v \frac{7}{2}[523]$ & $6^{+}\left(1^{+}\right)$ & 0.31 & 0 & 10.992 & $\left(5^{+}, 6^{+}, 4^{+}\right)$ & 0 & $11.130^{\#}$ & $2.6 \mathrm{~s}$ \\
\hline & $\pi \frac{5}{2}[532] \otimes \nu \frac{1}{2}[411]$ & $2^{-}\left(3^{-}\right)$ & 0.32 & 29 & & & & & \\
\hline \multirow[t]{2}{*}{${ }^{132} \mathrm{Eu}_{69}$} & $\pi \frac{3}{2}[411] \otimes \nu \frac{7}{2}[523]$ & $5^{-}\left(2^{-}\right)$ & 0.31 & 0 & 12.777 & & 0 & $12.740^{\#}$ & $100^{\#} \mathrm{~ms}$ \\
\hline & $\pi \frac{3}{2}[411] \otimes \nu \frac{1}{2}[411]$ & $1^{+}\left(2^{+}\right)$ & 0.32 & 28 & & & & & \\
\hline
\end{tabular}

${ }^{\mathrm{a} 130} \mathrm{Eu}$ decays by the proton emission [22].

the atomic mass difference between the ground states of the mother and the daughter is given as the $\beta$-decay energy, $Q_{\mathrm{EC}}=M(A, Z)-M(A, Z-1)$. We have calculated such energies with adjusted pairing strengths, reproducing well the experimental data [19] (see Tables I and II).

The properties of drip-line nuclei at shell closures are extremely interesting. Across the doubly magic nucleus, ${ }^{100} \mathrm{Sn}$, in Fig. 1, calculations show that there are low-lying prolate $9^{+} 2$ qp states with the configuration of $\pi 9 / 2^{+}[404] \otimes$ $v 9 / 2^{+}[404]$ in odd-odd ${ }^{110,112}$ I and ${ }^{112,114}$ Cs. Around the next proton closed shell of $Z=82$, there exist many lowlying high-spin 2qp states in $A \sim 190$ odd-odd nuclei. It is interesting that the high-spin states in the same nucleus can have very different shapes: prolate and oblate. Their details are predicted in Table III. In ${ }^{194,196,198} \mathrm{Bi},{ }^{198,200,202} \mathrm{At}$, and $202,204,206 \mathrm{Fr}$, an experiment [29] reported possible $10^{-}$and $7^{+}$ isomers with the assigned configurations of $\pi h_{9 / 2} \otimes \nu i_{13 / 2}$ and $\pi h_{9 / 2} \otimes v f_{5 / 2}$, respectively. In the present configurationconstrained calculations, only the deformed high-spin states can be treated. In ${ }^{192} \mathrm{At}$, a $9^{-}$or $10^{-}$isomer was observed with an oblate shape suggested [30]. However, our calculations give oblate $10^{+}$and prolate $10^{-}$states (see Table III).

In the transuranium region, the proton drip line is far from being reached experimentally. As shown in Fig. 1, high-spin isomeric states can occur in proton-rich transuranium nuclei.
The nuclei are calculated to have rather rigid prolate shapes with $\beta_{2} \approx 0.23$. Recently, a $T_{1 / 2}=3.6 \mathrm{~m}, 5^{-}$state (considered to be the ground state) and a $T_{1 / 2}=2.9 \mathrm{~m}, 1^{-}$isomer were observed in ${ }^{236} \mathrm{Am}$ [31]. These two states were suggested to be the GM doublet of the configuration $\pi 5 / 2^{-}[523] \otimes \nu 5 / 2^{+}[633]$ that results in the $5^{-}$and $1^{-}\left(K^{\pi}=0^{-}\right)$states [31]. Our calculations indicate that the $\pi 5 / 2^{+}[642] \otimes \nu 5 / 2^{+}[633]$ and $\pi 5 / 2^{-}[523] \otimes \nu 5 / 2^{+}[633]$ couplings form the two lowest configurations in ${ }^{236} \mathrm{Am}$.

In the most proton-rich nuclei, an unbound proton can be emitted directly from ground or isomeric states. In the high-spin 2qp states of odd-odd nuclei, however, there would be an additional spin inhibition (e.g., centrifugal barrier and interaction between the odd nucleons) leading to longer lifetimes. In Ref. [32], it has been pointed out that different $K$ values in the wave functions of the mother and the daughter can result in an additional $K$ hindrance of the proton decay. These would provide the possibility to extend the nuclide territory further beyond the proton-drip line. For example, a low-spin ground state might decay too fast $(\sim 10 \mathrm{~ns}$, say) for experimental observation, while a high-spin isomer in the same nuclide could be much longer lived. Such a scenario has already been suggested to occur in ${ }^{159} \mathrm{Re}$ and some other odd- $Z$ nuclides [33], presenting a remarkable nuclear-structure situation. 
TABLE III. Predicted low-lying high-spin states in $A \sim 190$ odd-odd nuclei around the $Z=82$ shell closure.

\begin{tabular}{|c|c|c|c|c|c|c|}
\hline Nuclei & Configurations & $J^{\pi}$ & $\beta_{2}$ & $\gamma$ & $\beta_{4}$ & $E_{x}(\mathrm{MeV})$ \\
\hline${ }^{180} \mathrm{Tl}_{99}$ & $\pi \frac{11}{2}^{-}[505] \otimes v \frac{7}{2}^{+}[633]$ & $9^{-}$ & 0.24 & $12^{\circ}$ & 0.030 & 0.37 \\
\hline \multirow[t]{3}{*}{${ }^{182} \mathrm{Tl}_{101}$} & $\pi \frac{11}{2}^{-}[505] \otimes v \frac{5}{2}^{-}[512]$ & $8^{+}$ & 0.24 & $13^{\circ}$ & 0.017 & 0.21 \\
\hline & $\pi \frac{11}{2}^{-}[505] \otimes v \frac{7}{2}^{+}[633]$ & $9^{-}$ & 0.24 & $0^{\circ}$ & 0.017 & 0.18 \\
\hline & $\pi \frac{9}{2}^{-}[505] \otimes v \frac{11}{2}^{+}[615]$ & $10^{-}$ & 0.16 & $60^{\circ}$ & 0.003 & 0.51 \\
\hline \multirow[t]{3}{*}{${ }^{184} \mathrm{Tl}_{103}$} & $\pi \frac{11}{2}^{-}[505] \otimes v \frac{5}{2}^{-}[512]$ & $8^{+}$ & 0.24 & $13^{\circ}$ & 0.005 & 0.20 \\
\hline & $\pi \frac{11}{2}^{-}[505] \otimes v \frac{7}{2}^{+}[633]$ & $9^{-}$ & 0.23 & $15^{\circ}$ & 0.003 & 0.31 \\
\hline & $\pi \frac{9}{2}^{-}[505] \otimes v \frac{11}{2}^{+}[615]$ & $10^{-}$ & 0.16 & $60^{\circ}$ & 0.002 & 0.47 \\
\hline${ }^{182} \mathrm{Bi}_{99}$ & $\pi \frac{11}{2}^{-}[505] \otimes v \frac{7}{2}^{+}[633]$ & $9^{-}$ & 0.26 & $13^{\circ}$ & 0.039 & 0.03 \\
\hline \multirow[t]{2}{*}{${ }^{184} \mathrm{Bi}_{101}$} & $\pi \frac{11}{2}^{-}[505] \otimes v \frac{5}{2}^{-}[512]$ & $8^{+}$ & 0.26 & $17^{\circ}$ & 0.026 & 0.20 \\
\hline & $\pi \frac{11}{2}^{-}[505] \otimes v \frac{7}{2}^{+}[633]$ & $9^{-}$ & 0.25 & $10^{\circ}$ & 0.025 & 0.21 \\
\hline \multirow[t]{2}{*}{${ }^{186} \mathrm{Bi}_{103}$} & $\pi \frac{11}{2}^{-}[505] \otimes \nu \frac{5}{2}^{-}[512]$ & $8^{+}$ & 0.26 & $3^{\circ}$ & 0.011 & 0.20 \\
\hline & $\pi \frac{11}{2}^{-}[505] \otimes v \frac{7}{2}^{+}[633]$ & $9^{-}$ & 0.24 & $16^{\circ}$ & 0.010 & 0.38 \\
\hline \multirow[t]{3}{*}{${ }^{188} \mathrm{Bi}_{105}$} & $\pi \frac{11}{2}^{-}[505] \otimes v \frac{7}{2}^{-}[514]$ & $9^{+}$ & 0.25 & $13^{\circ}$ & -0.003 & 0.00 \\
\hline & $\pi \frac{9}{2}^{-}[505] \otimes v \frac{9}{2}^{+}[624]$ & $9^{-}$ & 0.19 & $60^{\circ}$ & 0.007 & 0.33 \\
\hline & $\pi \frac{13}{2}^{+}[606] \otimes v \frac{9}{2}^{+}[624]$ & $11^{+}$ & 0.21 & $60^{\circ}$ & 0.012 & 0.27 \\
\hline \multirow[t]{3}{*}{${ }^{190} \mathrm{Bi}_{107}$} & $\pi \frac{11}{2}^{-}[505] \otimes v \frac{9}{2}^{+}[624]$ & $10^{-}$ & 0.23 & $15^{\circ}$ & -0.015 & 0.26 \\
\hline & $\pi \frac{9}{2}^{-}[505] \otimes v \frac{7}{2}^{+}[633]$ & $8^{-}$ & 0.20 & $60^{\circ}$ & 0.003 & 0.19 \\
\hline & $\pi \frac{13}{2}^{+}[606] \otimes v \frac{7}{2}^{+}[633]$ & $10^{+}$ & 0.21 & $60^{\circ}$ & 0.008 & 0.12 \\
\hline \multirow[t]{3}{*}{${ }^{190} \mathrm{At}_{105}$} & $\pi \frac{11}{2}^{-}[505] \otimes v \frac{7}{2}^{-}[514]$ & $9^{+}$ & 0.26 & $12^{\circ}$ & 0.004 & 0.44 \\
\hline & $\pi \frac{11}{2}^{-}[505] \otimes v \frac{7}{2}^{+}[633]$ & $9^{-}$ & 0.25 & $16^{\circ}$ & 0.003 & 0.90 \\
\hline & $\pi \frac{13}{2}^{+}[606] \otimes v \frac{9}{2}^{+}[624]$ & $11^{+}$ & 0.22 & $60^{\circ}$ & 0.013 & 0.92 \\
\hline \multirow[t]{2}{*}{${ }^{192} \mathrm{At}_{107}$} & $\pi \frac{11}{2}^{-}[505] \otimes v \frac{9}{2}^{+}[624]$ & $10^{-}$ & 0.25 & $16^{\circ}$ & -0.009 & 0.52 \\
\hline & $\pi \frac{13}{2}^{+}[606] \otimes v \frac{7}{2}^{+}[633]$ & $10^{+}$ & 0.22 & $60^{\circ}$ & 0.009 & 0.37 \\
\hline \multirow[t]{2}{*}{${ }^{194} \mathrm{At}_{109}$} & $\pi \frac{13}{2}^{+}[606] \otimes v \frac{5}{2}^{+}[642]$ & $9^{+}$ & 0.22 & $57^{\circ}$ & 0.009 & 0.26 \\
\hline & $\pi \frac{13}{2}^{+}[606] \otimes v \frac{5}{2}^{-}[503]$ & $9^{-}$ & 0.22 & $60^{\circ}$ & -0.001 & 0.66 \\
\hline \multirow[t]{2}{*}{${ }^{196} \mathrm{At}_{111}$} & $\pi \frac{13}{2}^{+}[606] \otimes v \frac{5}{2}^{+}[642]$ & $9^{+}$ & 0.22 & $60^{\circ}$ & 0.005 & 0.31 \\
\hline & $\pi \frac{13}{2}^{+}[606] \otimes v \frac{5}{2}^{-}[503]$ & $9^{-}$ & 0.22 & $56^{\circ}$ & -0.002 & 0.43 \\
\hline \multirow[t]{2}{*}{${ }^{198} \mathrm{At}_{113}$} & $\pi \frac{13}{2}^{+}[606] \otimes v \frac{5}{2}^{+}[642]$ & $9^{+}$ & 0.20 & $60^{\circ}$ & -0.002 & 0.74 \\
\hline & $\pi \frac{13}{2}^{+}[606] \otimes v \frac{5}{2}^{-}[503]$ & $9^{-}$ & 0.21 & $53^{\circ}$ & -0.003 & 0.62 \\
\hline \multirow[t]{2}{*}{${ }^{198} \mathrm{Fr}_{111}$} & $\pi \frac{11}{2}^{+}[615] \otimes v \frac{5}{2}^{+}[642]$ & $8^{+}$ & 0.24 & $50^{\circ}$ & 0.013 & 0.21 \\
\hline & $\pi \frac{11}{2}^{+}[615] \otimes \nu \frac{5}{2}^{-}[503]$ & $8^{-}$ & 0.23 & $47^{\circ}$ & 0.005 & 0.23 \\
\hline \multirow[t]{2}{*}{${ }^{200} \operatorname{Fr}_{113}$} & $\pi \frac{11}{2}^{+}[615] \otimes v \frac{5}{2}^{+}[642]$ & $8^{+}$ & 0.22 & $47^{\circ}$ & 0.002 & 0.33 \\
\hline & $\pi \frac{11}{2}^{+}[615] \otimes v \frac{5}{2}^{-}[503]$ & $8^{-}$ & 0.23 & $49^{\circ}$ & -0.001 & 0.37 \\
\hline${ }^{202} \mathrm{Fr}_{115}$ & $\pi \frac{11}{2}^{+}[615] \otimes v \frac{3}{2}^{+}[651]$ & $7^{+}$ & 0.21 & $60^{\circ}$ & -0.011 & 0.76 \\
\hline${ }^{204} \mathrm{Fr}_{117}$ & $\pi \frac{11}{2}^{+}[615] \otimes v \frac{3}{2}^{+}[651]$ & $7^{+}$ & 0.19 & $60^{\circ}$ & -0.019 & 0.96 \\
\hline${ }^{204} \mathrm{Ac}_{115}$ & $\pi \frac{11}{2}^{+}[615] \otimes v \frac{3}{2}^{+}[651]$ & $7^{+}$ & 0.22 & $60^{\circ}$ & -0.010 & 0.68 \\
\hline${ }^{206} \mathrm{Ac}_{117}$ & $\pi \frac{11}{2}^{+}[615] \otimes v \frac{3}{2}^{+}[651]$ & $7^{+}$ & 0.20 & $60^{\circ}$ & -0.018 & 0.80 \\
\hline
\end{tabular}

\section{SUMMARY}

Using configuration-constrained PES calculations, we have investigated low-lying 2qp states in deformed odd-odd, protonrich nuclei at the limit of experimental accessibility. The mass $70,130,190$, and transuranium regions are favored for forming low-lying high-spin isomeric states. The present calculations are helpful in understanding the structures of observed isomers. In odd-odd nuclei, the energy differences between low-lying 2qp states can be very small, e.g., less than $100 \mathrm{keV}$. It is difficult for model calculations to reproduce such small differences, and hence the level ordering can be uncertain. Because of the strong spin inhibition in $\gamma$-ray transitions, some high-spin isomers can directly undergo $\beta^{+} / \mathrm{EC}$ decay into their 
corresponding even-even daughter nucleus. Also, because of spin selection in $\beta$ decay, a high-spin isomer can only decay into high-spin excited states of the even-even daughter, which results in a $\beta$-decay energy smaller than that for a low-spin state. Therefore, high-spin isomers can have longer overall lifetimes than low-spin ground states. Beyond the proton drip line, a similar situation can apply to proton decay. Possible high-spin isomers are predicted, which should be useful for the ongoing experimental endeavor to explore the limits of nuclear existence.

\section{ACKNOWLEDGMENTS}

This work was supported by the Chinese Major State Basic Research Development Program under Grant 2007CB815000; the Natural Science Foundation of China under Grants 10525520, 10475002, and 10375078; the Key Grant Project (Grant 305001) of the Education Ministry of China; the EPSRC (UK) and AWE plc; and the Swedish Science Research Council (V.R.). We also thank the PKU Computer Center where numerical calculations were done.
[1] R. F. Casten and B. M. Sherrill, Prog. Part. Nucl. Phys. 45, S171 (2000).

[2] M. Caamaño, P. M. Walker, P. H. Regan, M. Pfützner, Zs. Podolyák, J. Gerl, M. Hellström, P. Mayet, M. N. Mineva, A. Aprahamian et al., Eur. Phys. J. A 23, 201 (2005).

[3] R. S. Chakrawarthy, P. M. Walker, J. J. Ressler, E. F. Zganjar, G. C. Ball, M. B. Smith, A. N. Andreyev, S. F. Ashley, R. A. E. Austin, D. Bandyopadhyay et al., Phys. Rev. C 73, 024306 (2006).

[4] F. R. Xu, E. G. Zhao, R. Wyss, and P. M. Walker, Phys. Rev. Lett. 92, 252501 (2004).

[5] W. Królas, R. Grzywacz, K. P. Rykaczewski, J. C. Batchelder, C. R. Bingham, C. J. Gross, D. Fong, J. H. Hamilton, D. J. Hartley, J. K. Hwang et al., Phys. Rev. C 65, 031303(R) (2002).

[6] D. M. Cullen, M. P. Carpenter, C. N. Davids, A. M. Fletcher, S. J. Freeman, R. V. F. Janssens, F. G. Kondev, C. J. Lister, L. K. Pattison, D. Seweryniak et al., Phys. Lett. B529, 42 (2002).

[7] G. Audi, O. Bersillon, J. Blachot, and A. H. Wapstra, Nucl. Phys. A729, 3 (2003).

[8] C. J. Gallagher, Jr., and S. A. Moszkowski, Phys. Rev. 111, 1282 (1958).

[9] A. K. Jain, R. K. Sheline, D. M. Headly, P. C. Sood, D. G. Burke, I. Hrǐvnácová, J. Kvasil, D. Nosek, and R. W. Hoff, Rev. Mod. Phys. 70, 843 (1998).

[10] F. R. Xu, P. M. Walker, J. A. Sheikh, and R. Wyss, Phys. Lett. B435, 257 (1998).

[11] W. Nazarewicz, J. Dudek, R. Bengtsson, T. Bengtsson, and I. Ragnarsson, Nucl. Phys. A435, 397 (1985).

[12] W. Satuła, R. Wyss, and P. Magierski, Nucl. Phys. A578, 45 (1994).

[13] W. D. Myers and W. J. Swiatecki, Nucl. Phys. 81, 1 (1966).

[14] P. Möller and J. R. Nix, Nucl. Phys. A536, 20 (1992).

[15] F. R. Xu, R. Wyss, and P. M. Walker, Phys. Rev. C 60, 051301(R) (1999).

[16] A. Piechaczek, E. F. Zganjar, J. C. Batchelder, B. D. Macdonald, W. D. Kulp, S. D. Paul, R. Terry, and J. L. Wood, Phys. Rev. C 62, 054317 (2000).

[17] M. Karny, L. Batist, D. Jenkins, M. Kavatsyuk, O. Kavatsyuk, R. Kirchner, A. Korgul, E. Roeckl, and J. Zylicz, Phys. Rev. C 70, 014310 (2004).
[18] D. H. Perkins, Introduction to High Energy Physics (AddisonWesley, Menlo Park, 1987), p. 210.

[19] G. Audi, A. H. Wapstra, and C. Thibault, Nucl. Phys. A729, 337 (2003).

[20] J. M. D’Auria, J. W. Grüter, E. Hagberg, P. G. Hansen, J. C. Hardy, P. Hornshøj, B. Jonson, S. Mattsson, H. L. Ravn, P. Tidemand-Petersson et al., Nucl. Phys. A301, 397 (1978).

[21] S.-W. Xu, Z.-K. Li, Y.-X. Xie, Q.-Y. Pan, W.-X. Huang, X.-D. Wang, Y. Yu, Y.-B. Xing, N.-C. Shu, Y.-S. Chen et al., Phys. Rev. C 71, 054318 (2005).

[22] C. N. Davids, P. J. Woods, H. Mahmud, T. Davinson, A. Heinz, J. J. Ressler, K. Schmidt, D. Seweryniak, J. Shergur, A. A. Sonzogni et al., Phys. Rev. C 69, 011302(R) (2004).

[23] Y. Kojima, M. Asai, M. Shibata, K. Kawade, A. Taniguchi, A. Osa, M. Koizumi, and T. Sekine, Appl. Radiat. Isot. 56, 543 (2002).

[24] Xie Yuanxiang, Xu Shuwei, Li Zhankui, Yu Yong, Pan Qiangyan, Wang Chunfang, Zhang Tianmei, Long Guilu, and Li Yansong, Eur. Phys. J. A 5, 341 (1999).

[25] D. Barnéoud, J. Blachot, J. Genevey, A. Gizon, R. Béraud, R. Duffait, A. Emsallem, M. Meyer, N. Redon, and D. Rolando-Eugio, Z. Phys. A 330, 341 (1988).

[26] C. Ekström, S. Ingelman, G. Wannberg, M. Skarestad, and The ISOLDE Collaboration, Nucl. Phys. A292, 144 (1977).

[27] C. Thibault, F. Touchard, S. Büttgenbach, R. Klapisch, M. De Saint Simon, H. T. Duong, P. Jacquinot, P. Juncar, S. Liberman, P. Pillet et al., Nucl. Phys. A367, 1 (1981).

[28] S.-W. Xu (private communication).

[29] M. Huyse, P. Decrock, P. Dendooven, G. Reusen, P. Van Duppen, and J. Wauters, Phys. Rev. C 46, 1209 (1992).

[30] A. N. Andreyev, S. Antalic, D. Ackermann, S. Franchoo, F. P. Heßberger, S. Hofmann, M. Huyse, I. Kojouharov, B. Kindler, P. Kuusiniemi et al., Phys. Rev. C 73, 024317 (2006).

[31] M. Asai, M. Sakama, K. Tsukada, S. Ichikawa, H. Haba, I. Nishinaka, Y. Nagame, S. Goto, Y. Kojima, Y. Oura et al., Eur. Phys. J. A 23, 395 (2005).

[32] D. S. Delion, R. J. Liotta, and R. Wyss, Phys. Rev. C 68, 054603 (2003).

[33] D. T. Joss et al., Phys. Lett. B641, 34 (2006), and references therein. 